\title{
The era of precision medicine and its impact on nursing: paradigm shifts?
}

Luís Carlos Lopes Júnior'

ORCID: 0000-0002-2424-6510

'Universidade Federal do Espírito Santo. Vitória, Espirito Santo, Brazil.

How to cite this article:

Lopes Jr LC. The era of precision medicine and its impact on nursing: paradigm shifts? Rev Bras Enferm. 2021;74(5):e740501. https://doi.org/10.1590/0034-7167.2021740501

\section{INTRODUCTION}

The large investment and development of research in genomics and molecular biology in the last two decades, especially after the conclusion of the Human Genome Project (HGP), has generated many expectations regarding its impact on the transformation from the conventional medicine paradigm to the precision medicine paradigm ${ }^{(1-2)}$. The term precision medicine (PM) was used for the first time in 2011 in a report by the National Academy of Sciences of the United States, which proposed the bases for the elaboration of a new taxonomy of diseases based on molecular biology approaches. In the document, the term is used as a synonym for personalized medicine. The definition in the American document, Precision Medicine Initiative, is also not very different from the way personalized medicine has been conceived: "an emerging approach for disease treatment and prevention that takes into account individual variability in genes, environment, and lifestyle for each person"(2).

If, on the one hand, the completion of HGP enabled the sequencing of the human genome, substantially assisting in research and clinical practice in various areas of health, on the other hand, it did not meet all the propagated expectations that the benefits would be immediate and that would lead to the cure of several genetic diseases, in addition to there would be a great advance in biomedical research. In fact, there was a huge advance in terms of research, especially in the field of molecular biology, which allowed for a better understanding of the human genome, particularly regarding the structure and functioning of the genome, which culminated in the complete sequencing of this structure for the human species $^{(1-2)}$. However, the researchers concluded that, in isolation, genetic mapping and gene identification (structural genomics) would not explain most of the biological mechanisms, frustrating the initial expectation of definitively unveiling the causes of diseases and their ways of prevention. Thus, the challenge became to find possible correlations between the structure and function of each gene (functional genomics), which started the so-called "post-genomic era" (PGE) ${ }^{(1-2)}$. The PGE started two decades ago and, since then, sequencing equipment and techniques have evolved rapidly, in order to lower the cost of analysis and drastically reduce the time required for sequencing a complete genome ${ }^{(1)}$. For instance, with the launch of the second generation of sequencing in 2008, known as Next Generation Sequencing (NGS), a reduction in the cost of genome mapping and its introduction into clinical practice was made possible ${ }^{(1)}$. The new sequencers provide a fast, low-cost platform, far surpassing in efficiency those traditional sequencing technologies developed in the 1970s. The great advance offered by NGS is its ability to produce and process a huge volume of data in an increasingly fast, cost-effective and accurate way, allowing the understanding of the disease at the molecular level, increasing the effectiveness of diagnosis and treatment of various complex multifactorial diseases ${ }^{(1)}$.

Following the 1) data, 2) information and 3) knowledge flow, researchers around the world have been striving to bring the advances generated from 
the laboratory bench to patients' beds. Information technology is an indispensable tool in PM with regard to the large volume of data, so that there is a translation of knowledge to clinical practice $^{(1)}$. In this context, based on knowledge of structural genomics, techniques were promoted and perfected that allowed the advancement of research related to functional genomics, which became part of the so-called "omics" sciences - among the main ones are transcriptomics, proteomics, epigenomics and the metabolomics. These new approaches aim to understand changes in the functioning of the genome at different stages of development and under different environmental conditions, in order to provide a better understanding at the molecular level ${ }^{(1-2)}$. As genomic data, they are decisive for the understanding of various diseases and drug effects on physiological systems. The gap between genotype and phenotype can be studied through the characterization of different omics levels, including intermediate levels: transcriptome (RNA sequences and levels), proteome (the set of proteins present in the sample), metabolome (the set of metabolites); in addition to studies of non-coding regions of DNA, rich in microRNAs and gene expression regulatory sequences ${ }^{(1-2)}$. In summary, PGE was characterized by the awareness of a scenario of greater complexity than expected regarding the cellular mechanisms of gene information and regulation of its expression to help answer complex questions not yet elucidated with HGP.

In 2014, the Prime Minister of the United Kingdom started the 100,000 Genomes Project, with the aim of sequencing 100,000 genomes of individuals from the National Health Services (NHS), mainly in the search for biomarkers for cancer and rare genetic diseases. In 2015, in the United States, Barack Obama, President of the United States, announced to Congress the launch of a PM program with a budget of 215 million dollars, in which the genomes of $1,000,000,000$ people would be sequenced, with the promise to become a milestone in the transformation of American medicine. In 2016, China launched a 15-year project with an investment of 9.2 billion dollars in PM, aiming to make the country a global leader in the field ${ }^{(2-3)}$. In Brazil, genomic science and technology have been progressively incorporated into biomedical research, epidemiology (genome scan studies in population genetics) and clinical practice, especially in oncology. In 2015, the Brazilian Initiative on Precision Medicine (BIPMed) was launched in São Paulo, with the support of the São Paulo Research Support Foundation (FAPESP - Fundação de Amparo à Pesquisa do Estado de São Paulo), an initiative that brings together five research, innovation and dissemination centers (CEPIDs), with the objective of creating conditions to implement PM in Brazil(2). Among the available genetic tests are the genotyping of up to $1,000,000,000$ polymorphisms and the complete sequencing of the exome, seeking to list the genetic mutations associated with diseases and estimate genetic susceptibilities ${ }^{(2)}$.

PM promises to offer, based on the identification of patients' genetic characteristics, the precise medicine, in the exact dose and at the right time, making medicine more efficient and reducing the costs of medical care. While the paradigm of traditional medicine is characterized by the focus on treating the disease after the onset of symptoms, with diagnostic and treatment methods based on population averages - one-size-fits-all for disease prevention, diagnosis and treatment, the basis of PM lies in the recognition that different groups of individuals have specific genomic characteristics and that treatments should consider such differences ${ }^{(1,3)}$. Furthermore, PM aims to customize the treatment according to the biological characteristics of individuals or population subgroups. Furthermore, in the paradigm of traditional medicine, the physician's decision about which medication to prescribe is based on general information about treatment to patients. If the medication does not have a satisfactory response in some time (days/weeks), it can be changed. However, the "trial-and-error" approach can lead to adverse reactions, poor adherence to the treatment regimen and patient dissatisfaction. In turn, PM seeks to consider the differences in genotype and phenotype of individuals when making decisions about the best treatment. In this context, the main contribution of PM is to increase efficiency in clinical decision-making, by distinguishing, in advance, patients who are more likely to benefit from a given treatment from those with less chance. Thus, the possibility of incurring unnecessary treatment costs and the occurrence of adverse drug effects without achieving therapeutic success are reduced ${ }^{(1,3)}$.

Technologies related to PM contribute to making the Research \& Development (R\&D) process more efficient, either by reducing the time it takes to develop a product or by increasing its success rate. This approach presupposes the integration of several areas of knowledge, however it can be said that the PM R\&D is based mainly on three pillars of interdisciplinary and highly related knowledge: omics, bioinformatics and biomarkers ${ }^{(1,3)}$. Given what has been exposed so far, it is noted how immeasurable is the speed with which new genomic knowledge, with potential application to healthcare, is being discovered and has been transforming the healthcare mode $\mathrm{I}^{(1,3)}$. To meet the new demands for care, these advances need to be incorporated into professional nursing practice and, above all, into nursing care ${ }^{(3-4)}$. In order for patients and their families to fully benefit from the explosion of genomic knowledge, healthcare professionals, especially nurses, need to grasp the underlying principles of genomics that have been shaping all healthcare practice and care. These principles include, for example, the existence of alternative forms of the same gene (alleles) in the population; the occurrence of similar phenotypes arising from mutations and variations at different loci; the notion that certain diseases in families can arise from genetic variants that cause susceptibility to diseases amid genegene and gene-environment interactions; the different types of mutations; the possibility of performing prenatal diagnoses, pre-symptomatic tests and population screenings; and, finally, the promise of PM through gene-based therapies ${ }^{(3-4)}$.

Even in the face of new approaches to care, many healthcare professionals still consider genomics to be a medical specialty and not an integral part of their daily practice ${ }^{(3)}$. Therefore, the necessary emphasis on the professional training of nurses based on genomics will become an important requirement as the omics sciences will become part of routine care, no longer being exclusively an area of specialization ${ }^{(4-5)}$. Recently, in 2018, through COFEN Resolution $581 / 2018$, which updates, within the scope of the Cofen System/ Regional Nursing Councils, the procedures for Registration of Lato and Stricto Sensu Graduate Degrees granted to Nurses and approves the list of specialties, it is noteworthy that nursing in genetics and genomics was definitely recognized as a specialty ${ }^{(6)}$. In fact, one of 
the main challenges of nursing education in this century is the integration of the omics sciences in clinical practice ${ }^{(4-5,7)}$. For that, it will be necessary extensive continuing education of nurses, of professors of Undergraduate and Graduate Nursing Courses and the reformulation of more integrated curricular structures, as occurs in several North American institutions, combined with a broad process of permanent education in health to adequately prepare the nursing workforce in the era of $\mathrm{PM}^{(4-5,7)}$.

The realization of the PM paradigm is subject to three main challenges. The first is the scientific challenge of correlating the various clinical conditions, genotype information with phenotype information. The second challenge is that the greater impact of diagnostic genomic tests on clinical decision-making has led regulatory agencies to expand their oversight of this industry and, like any new regulation, it still requires time to adjust. The third challenge refers to technological incorporation and possible ethical impacts, as well as impacts on health systems. The incorporation of new technologies into medical practices does not just happen because of their clinical usefulness. Medicine transformation movements are influenced by political, historical, socioeconomic contexts in which different actors act, such as the pharmaceutical/biotechnology industry, researchers, healthcare professionals, politicians, patient associations, citizens, media and NGOs. Furthermore, the PM movement is quite controversial and has raised heated debates in the field of bioethics and the impacts on health systems. For the incorporation of new PM technologies, it is essential to undertake a cost-benefit assessment from an ethical perspective, which takes into account whether they will be accessible to all who can benefit and whether they will not aggravate health disparities.

\section{REFERENCES}

1. Khoury MJ, Galea S. Will Precision Medicine Improve Population Health? JAMA. 2016;316(13):1357-1358. https://doi.org/10.1001/ jama.2016.12260

2. Iriart JAB. Precision medicine/personalized medicine: a critical analysis of movements in the transformation of biomedicine in the early $21 \mathrm{st}$ century. Cad Saude Publica. 2019;35(3):e00153118. https://doi.org/10.1590/0102-311X00153118

3. Collins FS, Varmus H. A new initiative on precision medicine. N Engl J Med. 2015;372(9):793-5. https://doi.org/10.1056/NEJMp1500523

4. Lopes Jr LC. Medicina genômica e a Enfermagem. In: Associação Brasileira de Enfermagem, Bresciani HR, Martini JG, Mai LD, (Org.). PROENF Programa de Atualização em Enfermagem: Saúde do Adulto: Ciclo 16. Porto Alegre: Artmed Panamericana; 2021. p. 10-62. https://doi. org/10.5935/978-65-5848-215-4.C0003

5. Tonkin E, Calzone KA, Badzek L, Benjamin C, Middleton A, Patch C, et al. A roadmap for global acceleration of genomics integration across nursing. J Nurs Scholarsh. 2020;52(3):329-38. https://doi.org/10.1111/jnu.12552

6. Conselho Federal de Enfermagem. Resolução COFEN 581/2018. Atualiza no âmbito do Sistema Cofen/Conselhos Regionais de Enfermagem, os procedimentos para registro de título de pós-graduação lato e stricto sensu concedido a enfermeiros e lista as especialidades. Brasília: COFEN, 2018.

7. Regan M, Engler MB, Coleman B, Daack-Hirsch S, Calzone KA. Establishing the Genomic Knowledge Matrix for Nursing Science. J Nurs Scholarsh. 2019;51(1):50-57. https://doi.org/10.1111/jnu.12427 